

\title{
The proton spin sum rule chiral bag prediction, an update
}

\author{
H.Høgaasen \\ Fysisk Institutt, University of Oslo, \\ Blindern, N-0316 Oslo, Norway \\ and \\ F. Myhrer \\ Department of Physics and Astronomy, University of South Carolina, \\ Columbia, SC29208, USA
}

February 8, 2020

\begin{abstract}
.
We reevaluate a quark model prediction using the new QCD evolution function calculated to the 3 loop order and conclude that this model compares favorably with the new experimental results.
\end{abstract}


From the semi-leptonic decays of baryons the matrix element of the flavour changing parts of the axial currens are fairly well known [1, 2]. The first EMC measurements of the spin dependent proton structure function $g_{1}^{p}\left(x, Q^{2}\right)$ when integrated, gave information of the matrix element of the flavour singlet part of the axial current between proton states. The first experimental results [3]

$$
\Gamma_{p}\left(Q^{2}\right)=\int d x g_{1}^{p}\left(x, Q^{2}\right)=0.126 \pm 0.012(\text { stat }) \pm 0.026(\text { syst }),
$$

implied that the matrix element of the flavour singlet part of the axial current between proton states was compatible with zero within the error bars. Despite the large experimental errors, some models of the baryon structure would give results outside three standard deviations of the measured flavor singlet matrix element value. Since this was an unexpected result, it generated a lot of discussion, see a short review in Ref. [4], and forced us to reconsider our present model understanding of the baryonic structure. However, the Skyrmion model of Ref. [5] and the chiral bag model [6] was immediately recognized to give results that was within one standard deviation of the measured integral over $g_{1}^{p}\left(x, Q^{2}\right)$.

In the last two years there has been a lot of new important experimental results coming from deep inelastic polarized lepton scattering both on polarized proton, deuteron and helium targets [7, 8, 9, 10]. It is of interest to see how well these early model calculations compare with the new experiments when we also take into account recent progress in the calculation of QCD corrections. The spin structure function $g_{1}^{p}\left(x, Q^{2}\right)$ has now been measured at quite small values of $\mathrm{x}$, and a smooth extrapolation to $\mathrm{x}=0$ / gives a new value for the integrated proton spin structure function $\Gamma_{p}\left(Q^{2}\right)$ at $\bar{Q}^{2}=10 \mathrm{GeV}^{2} / \mathrm{c}^{2}$ [7]. 2]

$$
\Gamma_{p}\left(Q^{2}\right)=0.136 \pm 0.011 \pm 0.011
$$

This value is larger but compatible with the earlier result, eq.(11). To compare this new value with model prediction, requires a good knowledge of QCD radiative corrections and in particular the $\mathrm{SU}(3)$ flavor singlet $\mathrm{QCD}$ evolution function, which, it was argued, may strongly affect the quark model predictions [12]. Very recently this evolution function has been evaluated at the 3-loop level [13], and confirmed by Larin [14], who gives references to earlier works and who also refers to the order $\alpha^{3} \mathrm{QCD}$ radiative corrections calculations.

In the following we shall argue as if nature is $\mathrm{SU}(3)$ flavour symmetric, not because we believe that to be an extremely good approximation, but because frequently this assumption is used in extracting matrix elements of currents with a definite flavour from the experimental semi-leptonic decay data [1, 2]. Two main points are responsible for the present model understanding of the baryon structure $\Gamma_{p}\left(Q^{2}\right)$. The first is the role of the axial anomaly which we shall discuss in the chiral $\mathrm{SU}(3)$ limit. The second point concerns the baryon wave function itself and what we can learn from the other baryon spin observables.

To discuss the first point we present our model picture of the baryon structure. We shall assume that an effective Lorentz-scalar force confines the quarks to a finite region in

\footnotetext{
${ }^{1}$ See a recent discussion on this point by Close and Roberts 11 .

${ }^{2}$ The SM Collaboration have also evalated a "world average" [] at $Q^{2}=10 \mathrm{GeV}^{2}$ of

$$
\left.\Gamma_{p}\left(10 G e V^{2}\right)=0.142 \pm 0.008 \pm 0.011\right)
$$
}


space (which we shall take to be a spherical cavity in the rest system of the baryon) where they interact through the exchange of gluons. As the valence quarks are excluded from the QCD vacuum outside the cavity, they will polarize the region just outside the cavity in $\bar{q} q$ pairs in such a manner that the axial current carried by the valence quarks extends into the QCD vacuum surrounding the cavity by $\bar{q} q$ pairs carrying the quantum numbers $J^{P}=0^{-}$, i.e., the pairs are the effective Goldstone bosons of the QCD vacuum. For chiral symmetry to be a good symmetry, it is necessary (but not sufficent) that the effective Goldstone fields in the QCD vacuum couple to the axial currents of the quarks in the cavity such that the axial current is continuous in all space.

In the chiral $\mathrm{SU}(3)$ limit the valence quarks $(\mathrm{u}, \mathrm{d}, \mathrm{s})$ in the cavity are massless. The pseudoscalar Goldstone bosons surrounding the quark confinement region, i.e., the pion, the $\eta_{8}$ and the kaon, are also massless. (This naturally imply that the $\bar{q} q$ content of the proton is mainly a large distance phenomenon.) On the other hand, the flavour singlet pseudoscalar, the $\eta^{\prime}$, is massiv in the chiral limit due to the $\mathrm{U}(1)$ anomaly. It is this consequence of the anomaly that we shall explicitly use to illustrate how a polarised $s \bar{s}$ cloud could exist around the "bare" nucleon (the nucleon's quark core), even if the "bare" nucleon contains no strange quarks. To illustrate this we use the following simplistic picture: Suppose for the sake of argument that we have a single confined u quark. Since we are in the chiral limit the axial current, which is carried by the u-quark inside the confinement cavity, has to be continued into the vacuum outside the cavity by massless pseudoscalar mesons, which would carry the flavour content of a $u \bar{u}$ state. This $u \bar{u}$ state can be written in terms of states that have well defined transformation properties under $\mathrm{SU}(3)$ flavour at the confinement "surface" where it changes into pseudoscalar mesons:

$$
u \bar{u}=1 / \sqrt{2} \pi^{0}+1 / \sqrt{3} \eta^{\prime}+1 / \sqrt{6} \eta_{8}
$$

If all pseudoscalars were massless, the $s \bar{s}$ content of $\eta_{8}$ and $\eta^{\prime}$ would cancel trivially, as is implied by this equality. However, the three pseudoscalar mesons have different masses, and therefore their Yukawa ranges beyond the cavity surface will be different. For example, even in the chiral limit the heavy $\eta^{\prime}$ (heavy due to the anomaly) and the massless $\eta_{8}$ would generate a strangeness content in the proton since the strength of the heavy $\eta^{\prime}$ field would decay exponentially outside the confinement "surface". Therefore, the "bare" nucleon containing only nonstrange quarks has a cloud of $s \bar{s}$ like states even in the chiral SU(3) limit as a consequence of the $\mathrm{U}(1)$ anomaly.

The second important point in these considerations is as follows: If one wants to compute matrix elements of the flavour singlet current, there is no reason to have confidence in the results if the model is not giving correct results for the matrix elements of the flavour octet currents which are responsible for weak semi-leptonic baryon decays. From the experience with the MIT bag model [15] we know that relativistic quarks reduce the neutron axial charge $g_{A}$ from $5 / 3$ towards a more realistic value. In addition, it is necessary to go beyond the usual zero order SU(6)-like baryon wave functions of an additive quark model. An additive quark model gives (in the chiral limit) for hyperon $\beta$-decays an amplitude ratio, $\mathrm{F} / \mathrm{D}=2 / 3$ like the non-relativistic quark model. The fallacy of the additive quark models becomes apparent for the baryon magnetic moments where these quark models give the wrong inequality, i.e., for the measured magnetic moments $\left|\mu\left(\Xi^{-}\right)\right|>|\mu(\Lambda)|[2]$. These "spin" problems of $F$ and $D$ and of the baryon magnetic moments are easily explained by invoking the same effective color magnetic spin-spin interaction that give the "bare" nucleon 
- "bare" $\Delta$ mass splitting [16, 17, 18]. This effective interaction introduces dynamic spin-spin correlations in the baryon wave functions that not only resolves the $\mathrm{F} / \mathrm{D}$ problem, $F / D \approx$ 0.57 , i.e. $<2 / 3$, but also easily explains why $\left|\mu\left(\Xi^{-}\right)\right|>|\mu(\Lambda)|$. This spin-spin correlation contributes to the neutron's $g_{A}$ and is important for the axial flavor-singlet charge [6, 19].

As should be evident from the above discussion, to make a sound model prediction for $\Gamma_{p}\left(Q^{2}\right)$ and $\Gamma_{n}\left(Q^{2}\right)$, it is necessary that the quark model reproduces the value for $g_{A}(=1.2573 \pm 0.0028)$ of the Bjorken sum rule as well as other baryon spin observables. Then one can discuss the model prediction for the flavor singlet part of the integrated proton spin structure function and the so-called "spin content of the proton". To be spesific we first define the relevant flavor $\mathrm{SU}(3)$ amplitudes [6, 20]. For $Q^{2} \rightarrow \infty$

$$
\Gamma_{p}\left(Q^{2} \rightarrow \infty\right)=<p \uparrow\left|\sum_{i} \frac{1}{2} \bar{\Psi}_{i} q_{i}^{2} \gamma_{5} \gamma_{3} \Psi_{i}\right| p \uparrow>=\frac{1}{6}\left[I_{0}+I_{8}+I_{3}\right]
$$

where $q_{i}$ is the charge of the i'th quark. The matrix element of the axial current operator between proton states on the r.h.s. of eq.(5) is evaluated at $Q^{2}=0$. To make contact with the experimental result of eq.(3) the axial current operator on the rigth-hand-side of eq.(5) should be renormalized at the point $Q^{2}=10 \mathrm{GeV}^{2} / \mathrm{c}^{2}$. In eq.(5) the $\mathrm{SU}(3)$ flavor singlet amplitude is

$$
I_{0}=\sqrt{\frac{2}{3}}<p \uparrow\left|\bar{\Psi} \gamma_{5} \gamma_{3} \lambda_{0} \Psi\right| p \uparrow>
$$

and the two flavor octet amplitudes are

$$
I_{8}=\frac{1}{2 \sqrt{3}}<p \uparrow\left|\bar{\Psi} \gamma_{5} \gamma_{3} \lambda_{8} \Psi\right| p \uparrow>
$$

and

$$
I_{3}=\frac{1}{2}<p \uparrow\left|\bar{\Psi} \gamma_{5} \gamma_{3} \lambda_{3} \Psi\right| p \uparrow>
$$

where $\lambda$ are the usual Gell-Mann matrices. In terms of the standard SU(3) amplitudes $F$ and $D$ for the baryon semi-leptonic decays, the two flavor octet amplitudes are

$$
I_{3}=\frac{1}{2}(F+D) \text { and } I_{8}=\frac{1}{2}(F-D / 3) .
$$

The correspondence between the $I_{i}$ 's and the commonly used $\Delta q$ 's are:

$$
\begin{aligned}
& \Delta u=\frac{1}{2} I_{0}+I_{8}+I_{3}, \\
& \Delta d=\frac{1}{2} I_{0}+I_{8}-I_{3}, \\
& \Delta s=\frac{1}{2} I_{0}-2 I_{8},
\end{aligned}
$$

and the "proton spin content" is:

$$
\Sigma=\Delta u+\Delta d+\Delta s=\frac{3}{2} I_{0}
$$

Since the two amplitudes $I_{3}$ and $I_{8}$ are matrix elements of conserved currents in the chiral $\mathrm{SU}(3)$ limit, their values are independent of the renormalization point. Therefore, models used to calculate $F$ and $D\left(I_{3}\right.$ and $\left.I_{8}\right)$ should have conserved axial octet currents in the chiral limit in order to be reliably used at the scale of the SMC experiment. This is a key requirement in any model prediction of the "proton spin content", a requirement which is not satisfied by the non-relativistic quark model, for example. A concrete example of a model satisfying the above condition is the chiral bag model, an extention of the 
MIT model. Calculations within this model are quite straigtforward, and give for many observables excellent insight and estimates of the relevant physics involved. This was (and is) for us a valid reason for our use of this model. One of the serious drawback of this and similar models is the sharp confinement "wall" (like a square well potential) and the associated translational invariance problem.

Before presenting our quark model results it is necessary to outline the radiative QCD corrections to this sum rule and the possible renormalization of the flavor-singlet axial current. The proton sum rule including the QCD corrections at the scale $Q^{2}$ have recently been calculated at the 3-loop level of perturbative QCD (for references see [14], and the later next order guestimates of Kataev [21]) and reads,

$$
\begin{aligned}
\Gamma_{p}\left(Q^{2}\right) & =\frac{1}{6}\left[1-\frac{\alpha\left(Q^{2}\right)}{\pi}-3.5833\left(\frac{\alpha\left(Q^{2}\right)}{\pi}\right)^{2}-20.2153\left(\frac{\alpha\left(Q^{2}\right)}{\pi}\right)^{3}-130\left(\frac{\alpha\left(Q^{2}\right)}{\pi}\right)^{4}\right] \\
& \left(I_{3}+I_{8}\right)+ \\
+ & \frac{1}{6}\left[1-\frac{\alpha\left(Q^{2}\right)}{\pi}-1.0959\left(\frac{\alpha\left(Q^{2}\right)}{\pi}\right)^{2}-3.7\left(\frac{\alpha\left(Q^{2}\right)}{\pi}\right)^{3}\right] Y\left(\alpha\left(\mu^{2}\right), \alpha\left(Q^{2}\right)\right) I_{0}\left(\mu^{2}\right) .
\end{aligned}
$$

As discussed, this expression assumes that the two flavour octet amplitudes, $I_{3}$ and $I_{8}$, are independent of the scale. On the other hand the scale dependence of the flavour singlet amplitude, $I_{0}\left(\mu^{2}\right)$, is made explicit in this equation. The effect of the anomalous dimensions of the flavor-singlet matrix elements are taken care of by the renormalization group (exponent) evolution function $Y\left(\alpha\left(\mu^{2}\right), \alpha\left(Q^{2}\right)\right)$, which very recently has been evaluated at the 3-loop level (in the lowest twist approximation), e.g. [14], and reads

$$
\ln Y\left(\alpha\left(\mu^{2}\right), \alpha\left(Q^{2}\right)\right)=\frac{2}{\pi} \int_{\alpha\left(\mu^{2}\right)}^{\alpha\left(Q^{2}\right)} d \alpha^{\prime} \frac{n_{f}-\gamma^{(1)} \frac{\alpha^{\prime}}{\pi}}{\beta_{0}+\frac{\beta_{1}}{4 \pi} \alpha^{\prime}+\frac{\beta_{2}}{(4 \pi)^{2}}\left(\alpha^{\prime}\right)^{2}} .
$$

where $\gamma^{(1)}=\left(1-\frac{71}{12}\right) n_{f}+\frac{1}{18} n_{f}^{2}$ and $\beta_{0}=11-2 n_{f} / 3, \beta_{1}=102-38 n_{f} / 3$, and $\beta_{2}=\frac{2857}{2}-\frac{5033}{18} n_{f}+\frac{325}{54} n_{f}^{2}$. The integrand is an increasing function of $\alpha^{\prime}$ in the region where the formula is valid.

To define the renormalization group-invariant (i.e., convention independent) nucleon matrix element of the singlet axial current [14, 23], we will write the evolution function as a product $Y\left(\alpha\left(\mu^{2}\right), \alpha\left(Q^{2}\right)\right)=Y\left(0, \alpha\left(Q^{2}\right)\right) Y\left(\alpha\left(\mu^{2}\right), 0\right)$. Then

$$
\Sigma_{i n v}=\exp \left(-\frac{1}{4 \pi} \int_{0}^{\alpha\left(\mu^{2}\right)} \mathrm{d} \alpha^{\prime} \frac{\gamma\left(\alpha^{\prime}\right)}{\beta\left(\alpha^{\prime}\right)}\right) \Sigma\left(\mu^{2}\right)=Y\left(\alpha\left(\mu^{2}\right), 0\right) \Sigma\left(\mu^{2}\right),
$$

and since $\alpha\left(Q^{2}\right)$ for $Q^{2}=10 \mathrm{GeV}^{2} / \mathrm{c}^{2}$ is small, we can use eq.(10) and write the expansion for $n_{f}=3$

$$
Y\left(0, \alpha\left(Q^{2}\right)\right) \approx 1+0.66667 \frac{\alpha\left(Q^{2}\right)}{\pi}+1.213\left(\frac{\alpha\left(Q^{2}\right)}{\pi}\right)^{2}+\cdots
$$


It then follows that the QCD corrections for the integrated spin-structure function are contained in QCD coefficients that are convention independent 14, 21]:

$$
\begin{array}{r}
\Gamma_{p}\left(Q^{2}\right)=\frac{1}{6}\left[1-\frac{\alpha\left(Q^{2}\right)}{\pi}-3.5833\left(\frac{\alpha\left(Q^{2}\right)}{\pi}\right)^{2}-20.2153\left(\frac{\alpha\left(Q^{2}\right)}{\pi}\right)^{3}-130\left(\frac{\alpha\left(Q^{2}\right)}{\pi}\right)^{4}\right]\left(I_{3}+I_{8}\right)+ \\
+\frac{1}{6}\left[1-\frac{\alpha\left(Q^{2}\right)}{3 \pi}-0.549\left(\frac{\alpha\left(Q^{2}\right)}{\pi}\right)^{2}-2\left(\frac{\alpha\left(Q^{2}\right)}{\pi}\right)^{3}\right] \frac{2}{3} \Sigma_{i n v} \cdot(13
\end{array}
$$

In the following we shall discuss the influence of the flavor singlet evolution function on the sum rule in eq.(9) and how the flavor singlet amplitude $I_{0, \text { model }}$, eq.(6) (or $\Sigma_{\text {model }}$ ) calculated in the usual spectroscopic quark models which include quark confinement, should be included in the two expressions, eqs.(9) and (13). Two extreme viewpoints regarding the influence of the flavor-singlet evolution function $Y\left(\alpha\left(\mu^{2}\right), \alpha\left(Q^{2}\right)\right)$ [12, 22] and [23] were discussed, and still is a matter of debate. One viewpoint argues that it is not inconceivable that non-perturbative effects could cause an evolution down to the very low spectroscopic scale $\mu^{2}$ such that $Y\left(\alpha\left(\mu^{2}\right), \alpha\left(Q^{2}\right)\right)$ is a very small number, a number which in itself could explain the EMC result [12, 22]. This scenario implies that the confinement quark model calculation predicts the matrix element of the flavour singlet axial current $I_{0}\left(\mu^{2}\right)$ in eq.(9). Furthermore, such an argument would lead to practically no constraint on a quark model calculation of $I_{0}$. In one of our previous works [20] we adopted this viewpoint, see e.g. Refs. [12, 24], and discussed the effects the known behaviour of the renormalization group exponent could have on the "proton spin content". This contrasts with our original attitude [6]: using models with confined quarks to compute the matrix elements appropriate for the Bjorken limit, we compute physical observable quantities, quantities which should be independent of the renormalization point and therefore should be identified with the renormalization group invariant quantities [23] or $\Sigma_{i n v}$ of eq.(11). This means only perturbative QCD corrections outlined in eq.(13) should be included in the sum rule. The reason being that any realistic quark confinement model (including chiral bag models) has built-in long distance effects, meaning soft gluonic corrections are to some extent included in the quark wave functions of these models. In addition, the chiral bag model also includes the "very soft" effective mesonic mediators of the longest range nuclear forces. These effective $\bar{q} q 0^{-}$ degrees of freedom are important for understanding properties of baryons. This is of course very different from the parton model approach where in the first appoximation the valence quarks are free non-interacting quarks with no gluonic content.

After these discussions we can present some numerical estimates of what is expected for the term $Y\left(\alpha\left(\mu^{2}\right), \alpha\left(Q^{2}\right)\right) I_{0}\left(\mu^{2}\right)$ of eq.(9) and compare with the $\Sigma_{\text {model }}$ value calculated in the chiral bag model where the pseudoscalar mesons are excluded from the bag. We use the values for $F$ and $D$ of the chiral bag model calculated in the chiral limit [20] including the two-body magnetic gluon exchange spin-spin correction: $F=0.455$ and $\vec{D}=0.795(F / D$ $=0.57$ ). This means that in eq.(9) $I_{3}+I_{8}=F+D / 3=0.72$. As values for $\alpha\left(Q^{2}\right)$ we use the ones corresponding to $\Lambda=240 \mathrm{MeV}$ [25] that runs within one standard deviation of data 
from the $Z_{0}$-mass to the $\tau$-mass. Then we find using the experimental values of eq.(3): [3

$$
Y\left(\alpha\left(\mu^{2}\right), \alpha\left(Q^{2}\right)\right) I_{0}\left(\mu^{2}\right)=0.194 \pm 0.073 \pm 0.073 .
$$

This result should be compared with the direct calculation of the chiral bag, which gave $I_{0, \text { model }}=0.22$ [6], corresponding to a "spin content" of $\Sigma_{\text {model }}=0.33$ when the pseudoscalar mesons were excluded from the interior of the bag. ft Early in the spin-crises period [3], this calculated value, $\Sigma_{\text {model }}=0.33$, was considered to be too large, which certainly, with the new experimental results [7] and the refined calculations of the radiative corrections, no longer is the case. The latest Ellis and Karliner [26] analysis gives $\Sigma\left(Q^{2}=10 \mathrm{GeV}^{2}\right)=0.31 \pm 0.07$ (when higher twist effects are ignored). Since $I_{0, \text { model }}$ is very close the the present value of eq.(14), there is in this model little room for the scenario with $Y$ being very small. However, as discussed above, since the quark wave function of the chiral bag model contains soft-gluon effects, we should and shall identify $\Sigma_{\text {model }}=0.33$ with $\Sigma_{i n v}$ of eq. (13) when we calculate $\Gamma_{p}\left(Q^{2}\right)$. The corresponding result for $\Gamma_{n}\left(Q^{2}\right)$ is obtained from the above by substituting $I_{8}-I_{3}=-0.53$ for $I_{8}+I_{3}=0.72$ in the same equation (13). In figures 1 and 2 we show both the model results using eq. (13) and the experimental data as a function of $\alpha\left(Q^{2}\right)$. We have not included any corrections from higher twists that would influence the results at low $Q^{2}$, the reason being that there is doubt both about the size as well as the sign of these corrections [27]. As we can see, the model is giving results that are very satisfactory when compared with the experiments on both the proton and the neutron targets.

In this model the so-called proton spin content has many components. One third comes from the spin of the three valence quarks, the remainder originates from the orbital angular momentum (of the relativistic quarks) and from the gluonic exchanges between the valence quarks which can be regarded as confined $\bar{q} q$ pairs [18]. Note that the $\mathrm{U}(1)$ anomaly is essential in reconciling the correct values of $\mathrm{F}$ and $\mathrm{D}$ with a value of $\Delta \Sigma<0.57$. In terms of the $\Delta q$ 's the model gives $\Delta u=0.83, \Delta d=-0.42$ and $\Delta s=-0.08$ as $Q^{2} \rightarrow \infty$.

We have also noted that a recent calculation by Narison, Shore and Veneziano based on QCD spectral sum rules give results [28] that are very similar to the ones we have presented. Our results were calculated in the chiral limit for the three quarks where $S U(3)_{f}$ by definition is a good symmetry group. When quarks and pseudoscalar mesons are given masses $\left(m_{u}=m_{d} \approx 10 \mathrm{MeV}\right.$ and $\left.m_{s} \approx 200 \mathrm{MeV}\right), S U(3)_{f}$ is broken, then what are the changes? The neutral pion is fairly light but both $\eta$ and $\eta^{\prime}$ become heavy. Due to their heavy masses, the influence of both of the two pseudoscalar $s \bar{s}$ carrying mesons are then suppressed. This leaves almost no hidden strangeness around the nucleons, as shown in an earlier calculation for nucleons [6]. However, the results for $\Gamma_{p, n}(\infty)$ hardly change at all!, meaning the chiral bag model with broken $S U(3)_{f}$ symmetry and almost no $s \bar{s}$ content can describe data as well as the $S U(3)_{f}$ symmetric chiral bag model above. From these results it follows that the experimental data of today do not necessarily imply that $\Delta s \neq 0$ but but could just as well mean that $S U(3)_{f}$ symmetry is broken. This specific example should underline the fact that the extraction of $\Delta s$ from the data on the basis of $S U(3)_{f}$ symmetry should be taken with some caution.

\footnotetext{
${ }^{3}$ If we use the "word average" value$$
Y\left(\alpha\left(\mu^{2}\right), \alpha\left(Q^{2}\right)\right) I_{0}\left(\mu^{2}\right)=0.213 \pm 0.053 \pm 0.073 .
$$

${ }^{4}$ In a version of the model where the pseudoscalar field is continuous through the bag surface (cloudy bag), $I_{0, \text { cloudy }}=0.38$ corresponding to $\Sigma_{\text {cloudy }}=0.57[6]$, the same value as in the Ellis and Jaffe sum rule.
} 
When a model is able to reproduce pre-EMC data on static properties of the baryons such as the axial charge, coupling strength of pions to the baryons, magnetic moments etc., and also give reasonable fits to the SMC data [7] in a new domain when the effect of the U(1) anomaly on the $\eta^{\prime}$ mass is taken into account, there is a coherence that give us confidence that we have some understanding of the rudimentary aspects of the nucleon structure.

Correspondence with E. Reya are gratefully acknowledged. This work is supported in part by NSF grant no. PHYS-9310124, a NATO travel grant and a grant from Norges Forskningsråd. 


\section{References}

[1] J.M. Gaillard and G. Sauvage, Annu. Rev. Nucl. Part. Sci., 34 (1984) 351.

[2] Particle Data Group, Phys. Rev., D50 (1994) 1173.

[3] J. Ashman et al.; EMC Coll., Phys. Letters B206 (1988) 364 ; and Nucl. Phys. B328 (1989) 1.

[4] E.Reya, Springer Lecture Notes in Physics 426 (1994) 175.

[5] S. Brodsky, J. Ellis and M. Karliner, Phys. Letters B206 (1988) 309;

[6] H. Høgaasen and F. Myhrer, Phys. Letters B214 (1988) 123.

[7] D. Adams et al., the SMC collaboration, Phys. Letters B329 (1994) 399.

[8] B. Adeva et al., Phys.Letters B302 (1993) 533.

[9] P.L. Anthony et al. Phys.Rev.Lett. 71 (1993) 959.

[10] Y.Terrien,Talk at The XII International Seminar on High Energy Physics Problems,Dubna,12-17 September 1994.

[11] F.E. Close and R.G. Roberts, Phys. Lett. B336 (1994) 257.

[12] R.L. Jaffe, Phys. Letters B193 (1987) 101.

[13] E.B.Zijlstra and W.L. van Neerven, Leiden preprint, INLO-PUB-3/93, to be publ. in Nucl. Phys. B417 (1994) 61.

[14] S.A. Larin, CERN-TH.7208/94 (1994) preprint; Phys. Letters B334 (1994) 192.

[15] T. DeGrand et al., Phys.Rev. D12 (1975) 2060 .

[16] K. Ushio and H. Konashi, Phys. Letters B135 (1984) 468; K. Ushio, Phys. Letters B158(1985) 71; Z. Phys. C30 (1986) 115.

[17] Kobzarev et al., Yad. Fiz. 43 (1986) 1252 [Sov. J. Nucl. Phys., 43 (1986) 803]

[18] H. Høgaasen and F. Myhrer, Phys. Rev. D37 (1988) 1950.

[19] F. Myhrer and A.W. Thomas, Phys. Rev. D38 (1988) 1633.

[20] H. Høgaasen and F. Myhrer, Z. Phys. C48 (1990) 295.

[21] A.L. Kataev, CERN preprint CERN-TH 7333/94 (1994).

[22] R.L. Jaffe and A. Manohar, Nucl. Phys. B337 (1990) 509.

[23] M. Glück and E. Reya, Z. Phys. C39 (1988) 569.

[24] J. Kunz, P.J. Mulders and S. Pollock, Phys. Letters B222 (1989) 481.

[25] G.Altarelli,CERN-TH.7246/1994 (1994).

[26] J. Ellis and M. Karliner, CERN-Th 7324/94 (1994).

[27] Ji, and Unrau, Phys. Letters B333 (1994) 228.

[28] S. Narison et al., CERN-TH 7223/94 (1994). 


\section{Figure Caption}

Figure 1: Comparison of the chiral bag model calculation, eq.(13), with experimental data of $\Gamma_{p}\left(Q^{2}\right)$. Systematic errors are heavy vertical lines, statistical errors are thin vertical lines. Data are plotted for reasonable values of $\alpha\left(Q^{2}\right)$.

Figure 2: Same as Figure 1 but for $\Gamma_{n}\left(Q^{2}\right)$. 
This figure "fig1-1.png" is available in "png" format from: http://arxiv.org/ps/hep-ph/9501414v1 
This figure "fig1-2.png" is available in "png" format from: http://arxiv.org/ps/hep-ph/9501414v1 